Check for updates

Cite this: Chem. Commun., 2019, 55,9448

Received 21st June 2019,

Accepted 12th July 2019

DOI: $10.1039 / c 9 c c 04768 a$

rsc.li/chemcomm

\section{Light controlled cell-to-cell adhesion and chemical communication in minimal synthetic cells†}

\author{
T. Chakraborty, $\ddagger^{a}$ S. M. Bartelt, $\ddagger^{a}$ J. Steinkühler, ${ }^{b}$ R. Dimova (D) ${ }^{b}$ and \\ S. V. Wegner (iD $* a$
}

\begin{abstract}
Decorating GUVs, used as minimal synthetic cell models, with photoswitchable proteins allows controlling the adhesion between them and their assembly into multicellular structures with light. Thereby, the chemical communication between a sender and a receiver GUV, which strongly depends on their spatial proximity, can also be photoregulated.
\end{abstract}

Cell-to-cell communication is a central function in life and is an important aspect to consider in the context of bottom-up synthetic biology, which aims to understand basic features of life through the construction of minimal synthetic cells from molecules in vitro. ${ }^{1-3}$ In local intercellular communication a sender cell releases a chemical signal that is perceived by a receiver cell. A prime example of such communication is observed in neurons, where neurotransmitters are released from the presynaptic neuron into the synaptic cleft and bind to receptors on the postsynaptic neuron resulting in transduction of a signal into the cell. As also evident from this example, only cells that are near one another can sense the signal, because chemical signals can only travel relatively short distances before they become too diluted. Therefore, cell to cell communication in minimal synthetic cells involves (i) controlling signal transduction from a sender to a receiver cell and (ii) building multicellular networks with defined spatial structures bringing sender and receiver cells in proximity.

Minimal synthetic cells with different communication modes have provided insight into how to use cell-to-cell communication to program the collective and multicellular behaviour in communities of minimal synthetic cells. For example, DNA-based communication has been used for information processing in consortia of synthetic cells, ${ }^{4,5}$ quorum sensing behaviour has been achieved with minimal synthetic cells through diffusive transcription factors ${ }^{6}$ and predatory behaviour through direct

\footnotetext{
${ }^{a}$ Max Planck Institute for Polymer Research, 55128, Mainz, Germany.

E-mail: wegners@mpip-mainz.mpg.de

${ }^{b}$ Department of Theory and Biosystems, Max Planck Institute of Colloids and Interfaces, 14476, Potsdam, Germany

$\dagger$ Electronic supplementary information (ESI) available. See DOI: 10.1039/c9cc04768a

\$ These authors contributed equally to the work.
}

contact and signal transduction has been implemented in synthetic cell communities. ${ }^{7}$ Likewise, chemical signals have been transduced from sender to receiver compartments per enzyme cascades and diffusible payload using membrane permeable signals or pore forming proteins such as $\alpha$-hemolysin., ${ }^{5,8-10}$ These examples showcase the general concepts of cell to cell communication and the potential of these in producing emerging properties.

The distance between the sender and receiver cells in a multicellular network is the second central aspect to consider in local cell-to-cell communication. The importance of spatial organization in different minimal synthetic cells has been highlighted in recent studies where the sender and receiver cells were placed in defined geometries using microfluidics or optical tweezers. ${ }^{11}$ The alternative bottom-up approach to selfassemble multicellular structures relies on specific adhesions between different cell mimics and mirrors the principles of tissue organisation in multicellular organisms. ${ }^{12}$ The possibility to trigger and dynamically alter adhesions in response to external stimuli such as metal ions, ${ }^{13}$ temperature and light makes it possible to spatiotemporally change these multicellular structures. Moreover, optically modulating the interaction of two membranes can also result in an increase in the membrane are ${ }^{14}$ and fusion. ${ }^{15,16}$ Controlling the spatial arrangement of sender and receiver cells by controlling the adhesions between them represents a powerful way to regulate local cell-to-cell communication.

In this study, we show how controlling the adhesion between sender and receiver cells using light can be used to also control local cell-to-cell communication (Fig. 1). By implementing photoswitchable adhesions between the cells, we can form multicellular assemblies on demand and disassemble them in the dark. Photoregulation with visible light is particularly attractive as it provides high spatiotemporal control, is non-invasive, and allows tuning interactions. ${ }^{17,18}$ The light responsive assembly of these consortia thereby also allows controlling local cell-to-cell communication since receiver cells in close proximity to sender cells will perceive the released chemical signal.

In our design, we used giant unilamellar vesicles (GUVs) as cell mimetic compartments due to their similarity to eukaryotic 


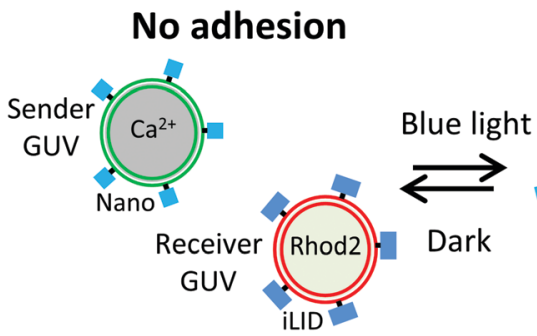

Adhesion
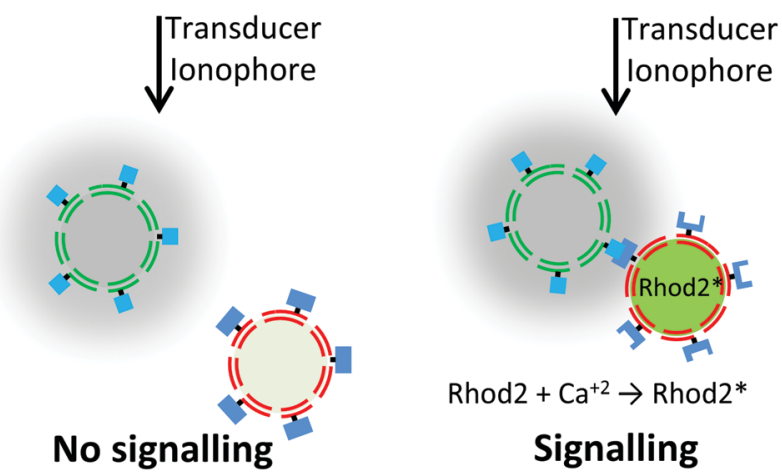

Fig. 1 The transmission of a signal from a sender GUV to a receiver GUV depends on their proximity, which can be controlled by GUV-GUV adhesion. The adhesion between sender and receiver GUVs can be reversibly controlled with blue light by decorating the GUVs with the proteins Nano and iLID, respectively, which bind to each other under blue light. When the sender and receiver GUVs adhere to each other and an ionophore is added as a transducer past the membranes, the chemical signal, $\mathrm{Ca}^{2+}$, can be communicated from the sender GUV to the receiver GUV, which becomes fluorescent upon Rhod2 binding to $\mathrm{Ca}^{2+}$

cells in terms of the size and membrane structure. As adhesion molecules, we employed the proteins iLID and Nano, which bind to each other under blue light $(480 \mathrm{~nm})$ and dissociate from each other in the dark. ${ }^{18}$ Such photoswitchable protein-protein interactions have been widely used in optogenetics to control diverse cell functions with light and are becoming valuable molecular building blocks in the context of bottom-up synthetic biology. ${ }^{17-19}$ Moreover, these photoswitchable proteins are biocompatible, have high specificity for their binding partners and operate in complex aqueous environments.

In the first step, the proteins iLID and Nano were immobilized on the outer membrane of two separate GUV populations containing through binding of the His-tags on the proteins to the $\mathrm{Ni}^{2+}$-nitrilotriacetic acid head group of the lipid (Fig. 2a), as previously reported. ${ }^{17,19}$ Each population of GUVs was fluorescently labelled with a membrane dye to differentiate them (iLIDGUV: DiD, Ex/Em: 644/665 nm, shown in red, and Nano-GUV: DiI, Ex/Em: 549/565 nm, shown in green). These fluorophores were chosen such that their excitation did not result in photoactivation of the blue light switchable iLID/Nano interaction.

The two GUV populations were mixed in a $1: 1$ ratio in the dark. GUVs of opposite types came into close proximity but did not interact strongly in the dark (Fig. 2a and Movie 1, ESI $\dagger$ ). Upon turning on blue light, which activated the iLID-Nano protein interaction, the GUVs adhered to each other within a few minutes, as evidenced by an increased overlap of the two vesicle membranes and the deformation of one of the vesicles. a)
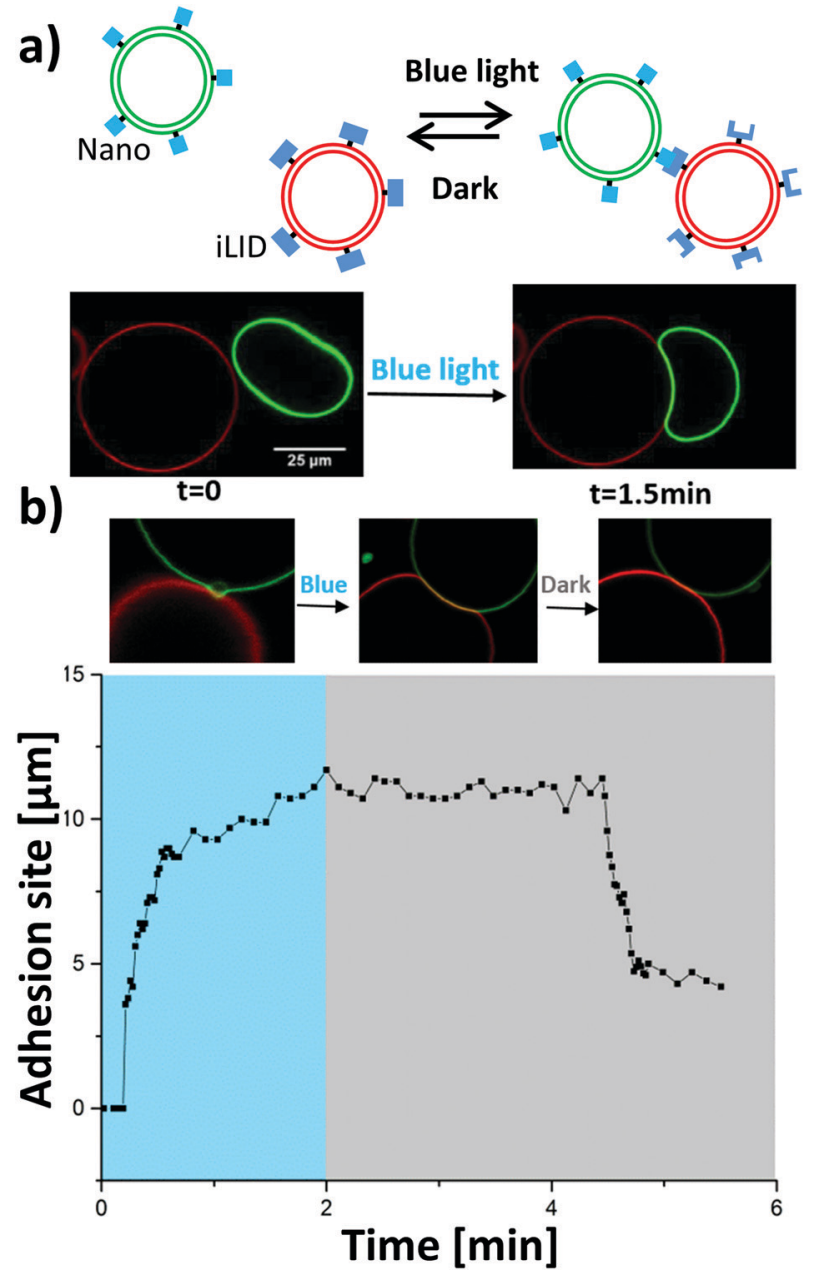

Fig. 2 Photoswitchable GUV-GUV adhesions. (a) Microscopy images of Nano (membrane in green) and iLID (membrane in red) functionalized GUVs, which interact with each other upon blue-light illumination, visible through the increase in the adhesion zone and the deformation of the deflated GUV (right). (b) Adhesion between Nano and iLID decorated GUVs is reversible in the dark. The GUV-GUV adhesion zone (arc length) over time under blue light (shaded in blue) and in the dark (shaded in grey) for the GUVs shown above.

One of the GUV populations was osmotically deflated so that upon adhesion the GUVs could deform and yield a large adhesion site. The adhesions between the GUVs were stable once formed over the duration of blue light illumination, without showing apparent fusion. Moreover, also in the bulk mixed population of the iLID and Nano functionalized GUVs large multi-GUV clusters were formed under blue light but not in the dark (Fig. S4a and c, ESI†).

The reversibility of adhesions is an important feature as it allows the disassembly of multicellular structures in response to changing stimulation and the separation of the sender and receiver cells at a desired time point. The GUV-GUV adhesions based on the iLID-Nano protein interactions are expected to be reversible, since the two proteins dissociate from each other in the dark. ${ }^{20}$ Indeed, when GUVs that adhered to each other under blue light were placed in the dark, the adhesion became less prominent up to complete detachment within a few minutes (Fig. $2 \mathrm{~b}$ and Movie 2, ESI $\dagger$ ). This was evident from a decrease in the overlap of the membranes of the two interacting GUVs and 
the complete (Fig. S1a, ESI $\dagger$ ) or partial (Fig. S1b, ESI $\dagger$ ) reversion of the bowl shaped deformation at the adhesion site. In cases where the deflated GUV deformed extensively into a bowl shape, the energy barrier became too high for the reversal of the deformation and these GUVs did not separate in the dark within a period of $20 \mathrm{~min}$ (Fig S1c, ESI $\dagger$ ). ${ }^{12}$

The kinetics of the blue light triggered adhesion and its reversion in the dark were analysed by measuring the length of the arc of its maximal cross section of the adhesion site between the two GUVs over time under changing illumination (Fig. 2b). GUV-GUV adhesions formed within the first minute of blue light activation, where two GUVs of opposite types coming into proximity expelling the water gap in between appears to be the rate limiting step and not the activation of the iLID protein with blue light, which happens within seconds of blue light illumination. ${ }^{18}$ Once the interaction partners iLID and Nano were in proximity so that the first contact occurred, the adhesion formed abruptly within a few seconds (Fig. $2 \mathrm{~b}$ and Fig. S2a, ESI $\dagger$ ). The final length of the adhesion site depended on the size of the two GUVs and the amount of the excess membrane of the deflated GUV. In the cases where the GUVGUV adhesions reversed in the dark, it was observed after a few minutes in one abrupt step (Fig. 2b and Fig. S2b, ESI†).

After having established the specific and photoswitchable GUV-GUV adhesions, we wanted to control cell-to-cell communication through the spatial organization of sender and receiver cells. We proposed that the exchange of a chemical signal would be more efficient when the sender and receiver GUVs adhere to each other under blue light (Fig. 1). Calcium ions play an important role in cellular signalling and have been implicated in the birth of cell sized lipid vesicles. ${ }^{21}$ In our design, the sender cells were GUVs containing $\mathrm{Ca}^{2+}(2 \mu \mathrm{M})$ as a chemical signal and were functionalized with Nano on their outer surfaces. The receiver cells were deflated GUVs loaded with the $\mathrm{Ca}^{2+}$ sensitive dye Rhod2 (500 nM), which becomes fluorescent upon $\mathrm{Ca}^{2+}$ binding and were functionalized with iLID on their outer surfaces. These GUVs were prepared by spontaneous swelling and loaded with the respective cargo by adding $\mathrm{Ca}^{2+}$ or Rhod2 into the rehydration buffer. Later, excess cargo on the outside of the GUVs was removed in repeated washing steps. For the $\mathrm{Ca}^{2+}$ to be transferred from the sender and receiver GUVs across the lipid membranes, we used the calcium selective ionophore, ionomycin $(830 \mathrm{nM})$, as a transducer. ${ }^{22}$ Indeed, when the ionophore was added to receiver GUVs in $\mathrm{Ca}^{2+}$ containing buffer, the Rhod2 fluorescence increased within a minute (Fig. S3, ESI $\dagger$ ), as detected using a plate reader.

To demonstrate that the signal transfer from the sender to the receiver GUVs depends on the adhesions between them, we mixed sender and receiver GUVs in equal amounts and incubated them for $1 \mathrm{~h}$ either in the dark or under blue light. Subsequently, we added the ionophore to these samples and measured the increase in the fluorescence of the $\mathrm{Ca}^{2+}$ sensitive dye Rhod2 (Ex/Em: 552/581 nm) using a plate reader. We observed that the fluorescence increased more for the sender/ receiver GUV mixture illuminated with blue light, where the GUVs form clusters, than the one kept in the dark, where the GUVs do
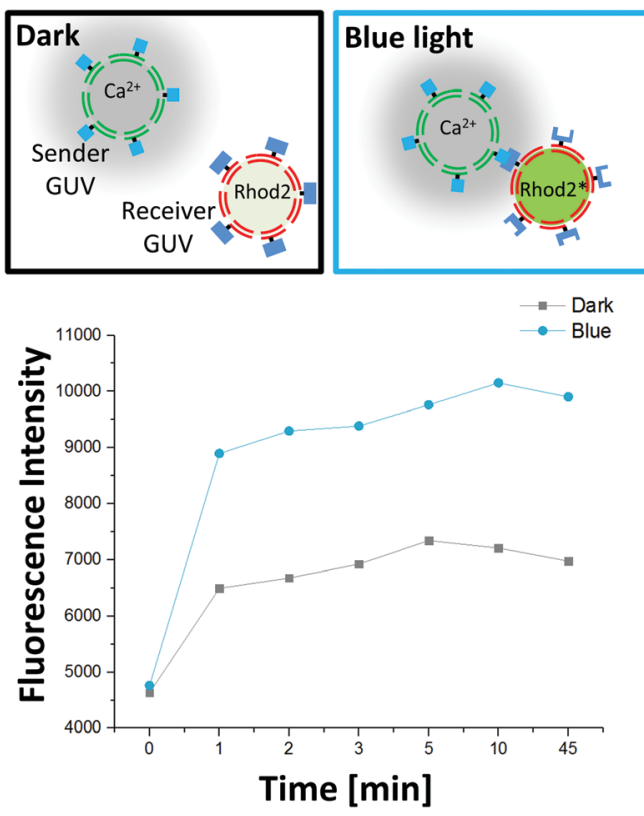

Fig. 3 Adhesion dependent GUV-GUV communication. Sender GUVs $\left(2 \mu \mathrm{M} \mathrm{Ca}^{2+}\right.$ ) and receiver GUVs (500 nM Rhod2) were incubated for $1 \mathrm{~h}$ either under blue light or in the dark before adding ionomycin and measuring the fluorescence of the $\mathrm{Ca}^{2+}$ sensitive dye Rhod2.

not interact (Fig. 3). This observation shows that the adhesion of a population of sender and receiver GUVs leads to a more effective transfer of the $\mathrm{Ca}^{2+}$ signal observed as an increase in the Rhod2 fluorescence. The increase in fluorescence was very rapid and complete within a minute. Moreover, the fluorescence was stable over longer periods indicating a stable transfer of the signal.

To gain further insight into how blue light dependent adhesions between sender and receiver GUVs alter $\mathrm{Ca}^{2+}$ signalling, we investigated this process for individual GUVs. For this purpose, sender GUVs with the membrane dye DiI (shown in green) and receiver GUVs with the membrane dye DiD (shown in red) were prepared. It should be noted that the fluorescent dyes DiI in the membranes of the sender GUVs and Rhod2 inside the receiver GUVs emit at similar wavelengths and were detected at once, yet, their distinct spatial localization made it possible to differentiate between them (Fig. 4a). We observed that sender and receiver GUVs adhered to each other under blue light (Fig. S4a, ESI $\dagger$ ) but not in the dark (Fig. S4c, ESI $\dagger$ ) after $1 \mathrm{~h}$ incubation. Further, the addition of ionomycin resulted in an increased Rhod2 fluorescence inside the receiver GUVs, which interacted with the sender GUVs under blue light (Fig. 4a and Fig. S4b, ESI $\dagger$ ). On the other hand, the increase in Rhod2 fluorescence was less prominent in non-adhering GUVs in the dark (Fig. S4d, ESI $\dagger$ ). These observations were quantified by measuring the mean fluorescence intensity inside the randomly picked receiver GUVs $(n=25)$ in the samples described above before and after adding the ionophore. To assure that the $\mathrm{Ca}^{2+}$ signal originates in the sender GUVs and not from the surrounding sender GUV buffer, receiver GUVs in the surrounding buffer of the sender GUVs were used as a control. This quantification showed that the mean Rhod2 intensity in the receiver GUVs increased significantly for samples kept under blue 
a)

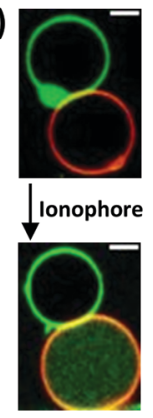

b)

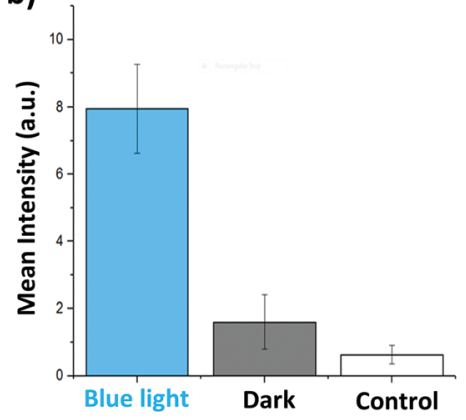

c)

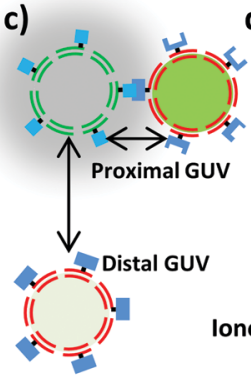

d)

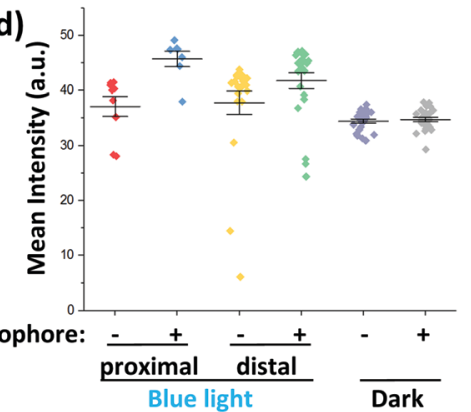

Fig. 4 Proximity controlled GUV-GUV communication. (a) Microscopy images of an interacting sender GUV $\left(\mathrm{Ca}^{2+}\right.$, membrane in green) and a receiver GUV (Rhod2 in green, membrane in red) before and after adding ionomycin. Rhod2 fluorescence increases inside the receiver. (b) Change in Rhod2 fluorescence inside the receiver GUVs in the presence of sender GUVs upon addition of ionomycin measured for individual GUVs in confocal microscopy images $(n=25)$. Buffer surrounding sender GUVs and no sender GUVs was used as a control. (c) Receiver GUVs in direct contact with sender GUVs (proximal GUVs) perceive the chemical signal more effectively than receiver GUVs at a further distance (distal GUV). (d) Rhod2 intensity in confocal microscopy images for proximal and distal GUVs under blue light and in the dark before $(-)$ and after $(+)$ adding ionomycin.

light but not for samples kept in the dark or the control sample after initiating the $\mathrm{Ca}^{2+}$ transfer by adding the ionophore (Fig. $4 \mathrm{~b}$ ). These results confirmed that successful chemical communication between sender and receiver GUVs strongly depends on their adhesion to each other.

As the distance between the sender and receiver GUVs plays a pivotal role in the transfer of the chemical signal, we investigated differences in response of receiver GUVs that were in direct contact (proximal) and were not interacting (distal $>10 \mu \mathrm{m}$ away) with sender GUVs under blue light illumination (Fig. 4c). We noted that the increase in the Rhod2 signal was larger for proximal receiver GUVs than that for distal GUVs after initiating the transfer of $\mathrm{Ca}^{2+}$ (Fig. 4d). Actually, the response of the distal receiver GUVs was comparable to the non-interacting GUVs in the dark. Similarly, when the response of individual GUVs was tracked over time, GUVs that formed direct contact with a sender GUV showed faster and stronger response than a GUV which was further away from the sender GUV once the ionophore was added (Fig S5, ESI $\dagger$ ).

In summary, we demonstrate how proximity between sender and receiver GUVs can be reversibly controlled by light using photoswitchable proteins iLID and Nano as adhesion molecules. Furthermore, the photoswitchable adhesions between sender and receiver GUVs provide a general approach to control their

proximity and hence local cell-to-cell communication in minimal synthetic cells. The reversibility and the high spatiotemporal control provided by the photoswitchable adhesions between the two different types of GUVs are important elements in assembling minimal synthetic cells housing different life-like processes into prototissues with high precision and to alter them dynamically. The molecular players in the study presented here are highly modular and can be implemented into other minimal synthetic cells. In particular, ionophores such as ionomycin are attractive alternatives to pore forming protein $\alpha$-hemolysin to achieve selective permeability for particular ions across lipid bilayers. As demonstrated in this study, cell-to-cell adhesion is a key factor to regulate chemical cell-to-cell communication also in the context of bottom-up synthetic biology.

This work is part of the MaxSynBio consortium, which is jointly funded by the Federal Ministry of Education and Research (BMBF) of Germany (FKZ 031A359L) and the Max Planck Society (MPG). Open Access funding provided by the Max Planck Society.

\section{Conflicts of interest}

There are no conflicts to declare.

\section{References}

1 S. Hennig, G. Rodel and K. Ostermann, J. Biol. Eng., 2015, 9, 13.

2 R. Lentini, N. Yeh Martin and S. S. Mansy, Curr. Opin. Chem. Biol., 2016, 34, 53.

3 Y. Elani, A. Gee, R. V. Law and O. Ces, Chem. Sci., 2013, 4, 3332.

4 A. Joesaar, S. Yang, B. Bogels, A. van der Linden, P. Pieters, B. Kumar, N. Dalchau, A. Phillips, S. Mann and T. F. A. de Greef, Nat. Nanotechnol., 2019, 14, 369.

5 K. P. Adamala, D. A. Martin-Alarcon, K. R. Guthrie-Honea and E. S. Boyden, Nat. Chem., 2017, 9, 431.

6 H. Niederholtmeyer, C. Chaggan and N. K. Devaraj, Nat. Commun., 2018, 9, 5027.

7 Y. Qiao, M. Li, R. Booth and S. Mann, Nat. Chem., 2017, 9, 110.

8 C. E. Hilburger, M. L. Jacobs, K. R. Lewis, J. A. Peruzzi and N. P. Kamat, ACS Synth. Biol., 2019, 8, 1223.

9 T. D. Tang, D. Cecchi, G. Fracasso, D. Accardi, A. CoutablePennarun, S. S. Mansy, A. W. Perriman, J. L. R. Anderson and S. Mann, ACS Synth. Biol., 2018, 7, 339.

10 V. Noireaux and A. Libchaber, Proc. Natl. Acad. Sci. U. S. A., 2004, 101, 17669.

11 G. Bolognesi, M. S. Friddin, A. Salehi-Reyhani, N. E. Barlow, N. J. Brooks, O. Ces and Y. Elani, Nat. Commun., 2018, 9, 1882.

12 S. Villringer, J. Madl, T. Sych, C. Manner, A. Imberty and W. Romer, Sci. Rep., 2018, 8, 1932.

13 S. Sun, M. Li, F. Dong, S. Wang, L. Tian and S. Mann, Small, 2016, 12, 1920.

14 V. N. Georgiev, A. Grafmuller, D. Bleger, S. Hecht, S. Kunstmann, S. Barbirz, R. Lipowsky and R. Dimova, Adv. Sci., 2018, 5, 1800432.

15 L. Kong, S. H. Askes, S. Bonnet, A. Kros and F. Campbell, Angew. Chem., 2016, 55, 1396.

16 Y. Suzuki, K. H. Nagai, A. Zinchenko and T. Hamada, Langmuir, 2017, 33, 2671.

17 S. M. Bartelt, J. Steinkuhler, R. Dimova and S. V. Wegner, Nano Lett., 2018, 18, 7268.

18 E. Chervyachkova and S. V. Wegner, ACS Synth. Biol., 2018, 7, 1817.

19 S. M. Bartelt, E. Chervyachkova, J. Steinkuhler, J. Ricken, R. Wieneke, R. Tampe, R. Dimova and S. V. Wegner, Chem. Commun., 2018, 54, 948.

20 G. Guntas, R. A. Hallett, S. P. Zimmerman, T. Williams, H. Yumerefendi, J. E. Bear and B. Kuhlman, Proc. Natl. Acad. Sci. U. S. A., 2015, 112, 112.

21 F. Tao and P. Yang, Sci. Rep., 2015, 5, 9839.

22 M. A. Kolber and D. H. Haynes, Biophys. J., 1981, 36, 369. 